\title{
PARENTS' PERCEPTION OF THEIR CHILD'S WEIGHT AND FACTORS RELATED TO CHILDHOOD OBESITY
}

\author{
J. Pascoe, M. Lee, C. Mcnicholas, W. Spears, M. Ebertino, G. Eberhart \\ Pediatrics, Wright State University, Dayton, OH, USA
}

Background and aims: Efforts to decrease childhood obesity may begin with accurate parental recognition of their overweight children. Using a written survey and body habitus silhouettes of children, this study examined parents' perceptions of their child's weight and factors related to childhood overweight (OW).

Methods: 285 parents of children between 2 years and 18 years being seen at six general pediatric practices that participate in the Southwestern Ohio Ambulatory Research Network (SOAR-Net) were asked to complete a survey that included demographics, varying children's body silhouettes (slender to OW) and whether their child was OW. Weight and height were measured to create a body mass index (BMI) for each study child.

Results: Study children's mean age was 8.4 (SD, 4.3) years and 34\% were OW (BMI $>=85$ th percentile). Compared to BMI measured during the office visit, sensitivity of parents identifying their child as "slghtly" or "very" OW was $42 \%$ (95\%CI:32-53\%).sensitivity of parents' selection of body silhouette for their child was similar $(43 \%)$. The specificity of parents' written or silhouette estimates was much higher $(>96 \%)$. Compared to parents of healthy weight (HW) children, parents of OW children were more likely to report that they themselves were overweight ( $61 \%$ vs $77 \%$, $\mathrm{p}=0.02)$, they "limited" their child's eating ( $38 \% 62 \%$, $\mathrm{p}<0.001)$ and annual household income was $<\$ 50,000(57 \%$ vs $79 \%, \mathrm{p}<0.001)$.

Conclusions: The specificity of parental estimates of their child's OW status was much higher than sensitivity. Childhood OW was related to a number of factors. 